\title{
Structure of Small Hydrogen Nanoclusters Containing Ortho-Molecules ${ }^{1}$
}

\author{
V. M. Akimov ${ }^{a}$, L. I. Kolesnikova ${ }^{a}$, L. Yu. Rusin ${ }^{a}$, M. B. Sevryuk ${ }^{a}$, and J. P. Toennies ${ }^{b}$ \\ ${ }^{a}$ Institute of Energy Problems of Chemical Physics, Russia Academy of Sciences, \\ Leninskii pr. 38, bldg. 2, Moscow, 119334 Russia \\ ${ }^{b}$ Max-Planck-Institut für Dynamik und Selbstorganisation, Bunsenstrasse 10, Göttingen, D-37073 Germany \\ e-mail:sevryuk@mccme.ru; rusin@chph.ras.ru
}

Received May 20, 2008

\begin{abstract}
Using the quantum mechanical path integral Monte Carlo method, we simulated parahydrogen clusters $(j=0)$ and clusters doped with several (up to 6) orthohydrogen molecules $(j=1$ ), with the total number of molecules ranging from 4 to 40 at temperatures of $1.5,3$, and $4.5 \mathrm{~K}$. Some energy parameters (including the chemical potentials) and spatial characteristics of the clusters are found. At a temperature of $1.5 \mathrm{~K}$, as the total number $N$ of molecules in the cluster increases, the chemical potential and the rotational energy of the clusters attain local minima at the same geometrically determined values of $N$ (the magic numbers). The ortho-molecules exhibit a larger probability (compared to the para-molecules) to reside in the central region of the cluster and a smaller probability to be located near its surface. This effect is enhanced as the number of orthohydrogen molecules in the cluster increases, the total number $N$ of molecules grows, or the temperature is lowered.
\end{abstract}

DOI: $10.1134 / \mathrm{S} 1990793109050078$

\section{INTRODUCTION AND DESCRIPTION OF THE MODEL}

After the transition of the clusters $\left(\text { para }-\mathrm{H}_{2}\right)_{13}$ and $\left(\text { para }-\mathrm{H}_{2}\right)_{18}$ to superfluid state at temperatures below $2 \mathrm{~K}$ was predicted theoretically in the work [1], the interest in small nanoclusters of molecular hydrogen and deuterium has increased considerably [2-12]. The current stage in studying such clusters started with the paper [13], where it was found by Raman spectroscopy that the clusters $\left(\text { para }-\mathrm{H}_{2}\right)_{13},\left(\text { para }-\mathrm{H}_{2}\right)_{33}$, and (para $\left.-\mathrm{H}_{2}\right)_{55}$ are more stable than the parahydrogen clusters $\left(\text { para }-\mathrm{H}_{2}\right)_{N}$ with the neighboring values of $N$. Lately, many theoretical investigations of nanoclusters of molecular hydrogen and deuterium were published [14-27]. In these works, the energy characteristics, the spatial structure, and the superfluidity properties of clusters $\left(\mathrm{H}_{2}\right)_{N}\left(\mathrm{D}_{2}\right)_{n}$ at temperatures no higher than several degrees Kelvin were determined by various (mainly stochastic) methods of quantum mechanical simulation.

The papers [1-27] studied almost exclusively clusters of parahydrogen and orthodeuterium, i.e. clusters of $\mathrm{H}_{2}$ and $\mathrm{D}_{2}$ molecules with even values of the rotational quantum number $j$ (actually, only the case $j=0$ was considered). The authors are aware of only two theoretical works where one explores clusters $\left(\mathrm{H}_{2}\right)_{N}\left(\mathrm{D}_{2}\right)_{n}$ including orthohydrogen or paradeuterium, i.e. $\mathrm{H}_{2}$ and $\mathrm{D}_{2}$ molecules with odd values of the rotational quantum number $j$. In the paper [7], the

\footnotetext{
${ }^{1}$ The article was translated by the authors.
}

clusters $\quad\left(\text { ortho }-\mathrm{D}_{2}\right)_{10}\left(\text { para }-\mathrm{D}_{2}\right)_{3}$, (ortho $\left.-\mathrm{D}_{2}\right)_{8}($ para $\left.\mathrm{D}_{2}\right)_{5}$, and $\left(\text { para }-\mathrm{D}_{2}\right)_{13}$ were simulated at temperatures from 1 to $3 \mathrm{~K}$. In the recent article [20], the cluster $\left(\text { para }-\mathrm{H}_{2}\right)_{24}\left(\text { ortho }-\mathrm{H}_{2}\right)_{1}$ was examined at temperatures of 0.5 and $1 \mathrm{~K}$. In both the works, one treated ortho- $\mathrm{H}_{2}$ and para $-\mathrm{D}_{2}$ molecules with $j=1$.

On the other hand, from the viewpoint of applications, clusters of orthohydrogen and paradeuterium are no less interesting than clusters of parahydrogen and orthodeuterium. Recall that normal hydrogen n$\mathrm{H}_{2}$ widely used in industry, power engineering, and scientific research and corresponding to thermodynamic equilibrium of the para- and ortho-components at room temperatures and higher is the mixture of $1 / 4$ of parahydrogen and $3 / 4$ of orthohydrogen whereas normal deuterium $n-D_{2}$ is the mixture of $2 / 3$ of orthodeuterium and $1 / 3$ of paradeuterium (e.g., see the review [28]). Some experimental works explored normal hydrogen clusters [29] and orthohydrogen clusters doped with an impurity molecule, for instance that of OCS [30] or $\mathrm{N}_{2} \mathrm{O}$ [31]. In crossed molecular beam studies of the dynamics of elementary processes involving $\mathrm{H}_{2}$ and $\mathrm{D}_{2}$, beams of normal hydrogen or deuterium are used very often. As an example, one can mention the papers [32-35] which considered the $\mathrm{F}+\mathrm{n}-\mathrm{H}_{2} \longrightarrow \mathrm{H}+\mathrm{HF}$ reaction and the articles $[36,37]$ devoted to elastic and inelastic $\mathrm{F}+\mathrm{n}-\mathrm{D}_{2}$ scattering. Small $\mathrm{H}_{2}$ and $\mathrm{D}_{2}$ clusters, even not very stable ones (first of all, dimers), containing molecules with 
odd $j$ values may play a noticeable role in such processes.

In the present paper, we have carried out systematic simulation of hydrogen nanoclusters (para $\left.-\mathrm{H}_{2}\right)_{N-m}\left(\text { ortho }-\mathrm{H}_{2}\right)_{m}$ in the ground state with the total number $N$ of molecules ranging from 4 to 40 and with the number of ortho-molecules $m=0,3,5$ at temperatures $T=1.5,3$, and $4.5 \mathrm{~K}$ using the quantum mechanical path integral Monte Carlo (PIMC) method. At a temperature of $1.5 \mathrm{~K}$ for some values of $N$, clusters containing $m=2,4$, and 6 hydrogen orthomolecules have also been simulated. The main principles of the PIMC method are expounded in detail in the works [7, 38-42]. Various modifications of this method were employed for simulating hydrogen and deuterium clusters in the papers $[1,3,4,7-10,15,16$, 18-20, 25-27].

The version of the PIMC method that we used is described in detail in the article [7] where it had been applied to studying the deuterium clusters (ortho $\left.-\mathrm{D}_{2}\right)_{10}\left(\text { para }-\mathrm{D}_{2}\right)_{3}$ and $\left(\text { ortho }-\mathrm{D}_{2}\right)_{8}\left(\text { para }-\mathrm{D}_{2}\right)_{5}$. Following [7], we assumed that all the hydrogen paramolecules are in the ground rotational state $j=0$ while all the ortho-molecules are in the state $j=1$. This is fully justified at temperatures of the order of a few degrees Kelvin. Two types of the rotational Hamiltonian basis had been considered in the paper [7] for a cluster containing $m$ molecules with $j=1$ : the full basis consisting of $3^{m}$ functions ("the A-basis") and an approximate basis ("the B-basis"). In order to obtain more accurate results, we performed calculations employing the full basis. However, since the number of functions in this basis grows exponentially as $m$ increases, the use of the A-basis is confined in practice with values $m \leq 6$.

Recall that the particles of the cluster are represented in the PIMC method by ring "polymers," each consisting of some number $M$ of "beads" [7, 38-42]. The larger $M$ is, the more accurate and simultaneously more laborious are the calculations and the smaller is a temperature $T$ that can be sampled. The case of classical particles corresponds to the value $M=1$. For temperatures $T=1.5,3$, and $4.5 \mathrm{~K}$, we used numbers $M$ lying in the intervals $741 \leq M \leq 765,679 \leq M \leq 684$, and $540 \leq M \leq 550$, respectively. Several tests showed that increasing $M$ further does not affect the calculation results. While determining any spatial characteristics of the cluster (e.g., the radial density profile or the distribution of the interparticle distances), one calculates the corresponding quantities (such as the distance to the center of mass or the pairwise distances) separately for each cluster "replica," i.e. for the collection of the $i$-th beads of all the polymers, $i=1, \ldots, M$.

The version of the PIMC method developed in the work [7] treats the intermolecular exchange effects in the Boltzmann statistics framework and, consequently, does not enable one to simulate superfluidity $[1,16,18-20,25-27,40]$. However, the energy char- acteristics and the spatial structure of small nanoclusters seem to depend slightly on the exchange effects [18].

Also following [7], we represented the isotropic component of the intermolecular $\mathrm{H}_{2}-\mathrm{H}_{2}$ interaction by the Lennard-Jones $(12,6)$ potential with the parameters $\varepsilon=34.2 \mathrm{~K}$ (the potential well depth) and $\sigma=2.96 \AA$ (the distance at which the potential vanishes). In the last 20 years, in most of the works devoted to hydrogen and deuterium nanoclusters [1$6,8,13-20,22-27]$, the more accurate SilveraGoldman potential $[28,43]$ or Buck potential [44, 45] have been employed. However, these two potentials yield qualitatively the same results as the LennardJones potential $[8,18]$. For classical clusters of $N \leq$ 1000 particles with a Lennard-Jones $(12,6)$ pairwise interaction potential, the configurations corresponding to the global minima of the potential energy are known [46] (for $N \leq 110$, such configurations are described in the paper [47]). We used these configurations scaled according to the value $\sigma=2.96 \AA$ as the initial ones while simulating the hydrogen clusters by the PIMC method. The anisotropic component of the intermolecular potential was assumed to be other than zero for the ortho- $\mathrm{H}_{2}-$ ortho- $\mathrm{H}_{2}$ pairs only and was represented as the quadrupole-quadrupole interaction [7].

\section{CALCULATION RESULTS}

Our calculations consisted mainly of the following seven series: simulation of parahydrogen clusters $\left(\text { para }-\mathrm{H}_{2}\right)_{N}$ at temperatures $T=1.5,3$, and $4.5 \mathrm{~K}$ for $4 \leq N \leq 40,6 \leq N \leq 40$, and $15 \leq N \leq 40$, respectively; simulation of clusters $\left(\text { para }-\mathrm{H}_{2}\right)_{N-3}\left(\text { ortho }-\mathrm{H}_{2}\right)_{3}$ with three orthohydrogen molecules at temperatures $T=$ $1.5,3$, and $4.5 \mathrm{~K}$ for $5 \leq N \leq 40,6 \leq N \leq 40$, and $15 \leq N \leq 40$, respectively; and, finally, simulation of clusters $\left(\text { para }-\mathrm{H}_{2}\right)_{N-5}\left(\text { ortho- } \mathrm{H}_{2}\right)_{5}$ with five orthohydrogen molecules at temperature $T=1.5 \mathrm{~K}$ for $5 \leq N \leq 40$. For $N$ values smaller than the indicated ones, clusters "evaporated," i.e. some molecules were leaving the cluster rapidly during the simulation by the PIMC method.

Figure 1 presents the total specific energies $E(N) / N$ of the clusters with three ortho-molecules (i.e. the total energies $E(N)$ per molecule) at temperatures $T=$ $1.5,3$, and $4.5 \mathrm{~K}$. The total specific energies of the pure parahydrogen clusters and of the hydrogen clusters with five ortho-molecules are almost undistinguishable in the scale of Fig. 1 from the total specific energies of the clusters $\left(\text { para }-\mathrm{H}_{2}\right)_{N-3}\left(\text { ortho }-\mathrm{H}_{2}\right)_{3}$ at the same total number $N$ of molecules and the same temperature.

In the framework of the so-called liquid drop model (e.g., see [48, 49]), the function $E=E(N)$ is fitted at a fixed temperature $T$ (and a fixed number $m$ of 
orthohydrogen molecules) by the three-parameter expression

$$
E(N) \approx A_{V} N+A_{s} N^{2 / 3}+A_{c} N^{1 / 3},
$$

where the terms $A_{V} N$ and $A_{S} N^{2 / 3}$ correspond to the "volume" and "surface" energies, respectively. One can interpret the quantity $-A_{V}$ as the bond energy of a molecule in a macroscopic hydrogen drop. Of course, such an interpretation is rather relative because liquid parahydrogen freezes at much higher temperatures ( $T=13.8 \mathrm{~K}$ at the triple point) than those sampled in this work, cf. the discussion in [50]. One sometimes uses the more general expression [51]

$$
E(N) \approx A_{\mathrm{v}} N+A_{s} N^{2 / 3}+A_{c} N^{1 / 3}+A_{d} .
$$

For each of the seven calculation series indicated above, we fitted the total energies $E(N)$ of the clusters by expression (1) by the least square method. The results are compiled in the table. As one sees from this table, each of the quantities $-A_{\mathrm{v}}, A_{s}$, and $-A_{c}$ is positive and increases as the temperature rises at a fixed number of ortho-molecules in the cluster and as the number of orthohydrogen molecules is raised at a fixed temperature. One expects that if the fitting is performed over wider $N$ ranges, the dependence of the coefficients $A_{\mathrm{v}}, A_{s}, A_{c}$ on the number $m=0,3,5$ of ortho-molecules in the cluster will vanish.

Another principal energy characteristic of a cluster (in addition to the total energy $E(N)$ ) is the chemical potential

$$
\mu(N)=E(N)-E(N-1),
$$

which is defined at the given temperature $T$ and the given number $m$ of orthohydrogen molecules. The $N$ values corresponding to the local minima of the function $\mu=\mu(N)$ are usually called the magic numbers: a cluster with a magic number of molecules is more stable than the clusters containing one more or one less molecule [14, 17-19, 21-24, 26]. More general concepts about cluster magic numbers are discussed, e.g., in the reviews $[52,53]$. The cluster chemical potentials $\mu(N)$ obtained in our calculations are presented in Figs. 2-4. For comparison, the same figures show the differences

$$
\theta(N)=U(N)-U(N-1),
$$

where $U(N)$ denotes the global minimum of the potential energy of a classical Lennard-Jones cluster of $N$ particles $[46,47]$ found under the condition that the potential well depth for the interaction of two particles is $\varepsilon=34.2 \mathrm{~K}$. One may call the quantity $\theta(N)$ the "classical chemical potential at zero absolute temperature."

At temperature $T=1.5 \mathrm{~K}$ (Fig. 2), the chemical potentials $\mu(N)$ in all the three cases we considered (parahydrogen clusters, hydrogen clusters with three ortho-molecules, and hydrogen clusters with five ortho-molecules) are very close and attain minima at

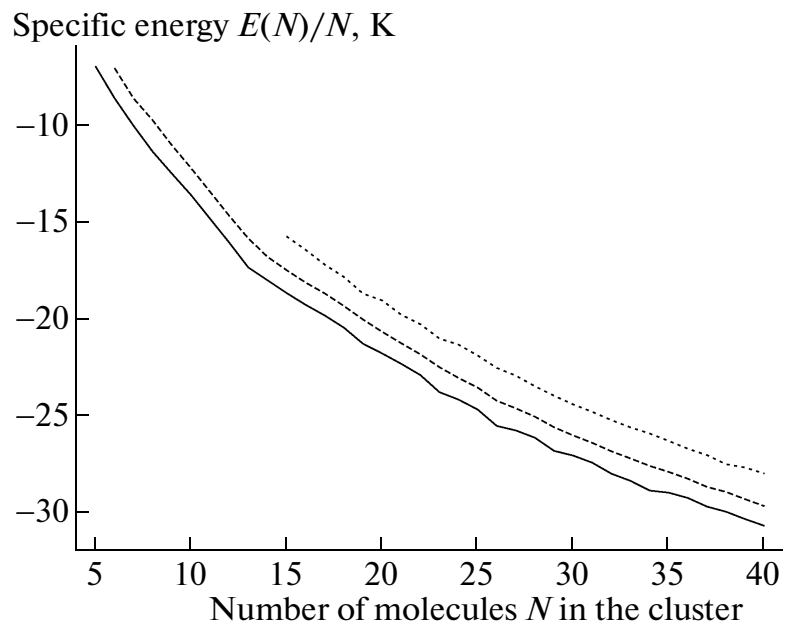

Fig. 1. The total specific energies of the clusters (para$\left.\mathrm{H}_{2}\right)_{N-3}\left(\text { ortho- }-\mathrm{H}_{2}\right)_{3}$ at temperatures $T=4.5,3$, and $1.5 \mathrm{~K}$ (from top to bottom). The standard deviations do not exceed a few centidegrees Kelvin.

the same values $N=13,19,23,26,29,32,34$, and 37 (which confirms the data of most of the preceding works [14, 17-19, 21-24, 26]). These magic numbers almost coincide with the positions of the local minima of the function $\theta=\theta(N)$ which are attained at $N=7$, $13,19,23,26,29,32,36$, and 38 in the range of $N$ values we sampled. Thus, the first six magic numbers $N=$ $13,19,23,26,29$, and 32 of hydrogen clusters at $T=$ $1.5 \mathrm{~K}$ are of a purely geometric origin and caused by the structure of the energy-minimal configurations (for a given number of particles) of classical Lennard-Jones clusters [46, 47]. The magic number $N=13$ which agrees with the experimental data [13] (as well as the magic number $N=55$ found experimentally [13]) corresponds to a closed icosahedral configuration [47]. The magic numbers for many other cluster characteristics known in the literature are also of a geometric nature (and connected with filling successively the cluster shells) $[52,53]$. Being based on the experimental results [13], one would expect the magic number $N=33$ rather than $N=32$ or 34 ; the reasons for this discrepancy are not quite clear. As the temperature $T$ is raised to $3 \mathrm{~K}$ (Fig. 3) and especially to $4.5 \mathrm{~K}$ (Fig. 4), the similarity between the chemical potentials (2) of the parahydrogen clusters and those of the hydrogen clusters with three ortho-molecules deteriorates, the minima of these potentials become much less pronounced, and the connection of the corresponding magic numbers with the local minima of function (3) disappears in essence (with the exception of the magic number $N=13$ at $T=3 \mathrm{~K}$ ).

Figure 5 presents the rotational energies $E_{\text {rot }}$ of the clusters with three and five orthohydrogen molecules. We defined the rotational Hamiltonian of the cluster to be the sum of the operator of the kinetic energy of molecular rotation and the operator of the anisotropic 
The fitting of the total energies of the clusters by expression (1). The values of the coefficients $A_{V}, A_{s}, A_{c}$ and the corresponding standard deviations are given in Kelvin

\begin{tabular}{|c|c|c|c|}
\hline Coefficients & $\begin{array}{l}\text { Values of the } \\
\text { coefficients }\end{array}$ & \multicolumn{2}{|c|}{ Standard deviations } \\
\hline \multicolumn{4}{|c|}{ Only para-molecules at $T=1.5 \mathrm{~K}$} \\
\hline$A_{V}$ & -76.8 & \pm 0.84 & $(1.1 \%)$ \\
\hline$A_{s}$ & 198 & \pm 3.8 & $(1.9 \%)$ \\
\hline$A_{c}$ & -133 & \pm 4.1 & $(3.1 \%)$ \\
\hline \multicolumn{4}{|c|}{ Only para-molecules at $T=3 \mathrm{~K}$} \\
\hline$A_{V}$ & -80.0 & \pm 1.1 & $(1.4 \%)$ \\
\hline$A_{s}$ & 218 & \pm 6.0 & $(2.8 \%)$ \\
\hline$A_{c}$ & -156 & \pm 7.8 & $(5.0 \%)$ \\
\hline \multicolumn{4}{|c|}{ Only para-molecules at $T=4.5 \mathrm{~K}$} \\
\hline$A_{V}$ & -82.3 & \pm 1.9 & $(2.3 \%)$ \\
\hline$A_{s}$ & 240 & \pm 11 & $(4.6 \%)$ \\
\hline$A_{c}$ & -185 & \pm 16 & $(8.6 \%)$ \\
\hline \multicolumn{4}{|c|}{ Three ortho-molecules at $T=1.5 \mathrm{~K}$} \\
\hline$A_{V}$ & -79.5 & \pm 0.99 & $(1.2 \%)$ \\
\hline$A_{s}$ & 211 & \pm 4.9 & $(2.3 \%)$ \\
\hline$A_{c}$ & -150 & \pm 6.0 & $(4.0 \%)$ \\
\hline \multicolumn{4}{|c|}{ Three ortho-molecules at $T=3 \mathrm{~K}$} \\
\hline$A_{V}$ & -80.3 & \pm 1.2 & $(1.4 \%)$ \\
\hline$A_{s}$ & 220 & \pm 6.2 & $(2.8 \%)$ \\
\hline$A_{c}$ & -159 & \pm 8.2 & $(5.1 \%)$ \\
\hline \multicolumn{4}{|c|}{ Three ortho-molecules at $T=4.5 \mathrm{~K}$} \\
\hline$A_{V}$ & -83.7 & \pm 2.0 & $(2.4 \%)$ \\
\hline$A_{s}$ & 248 & \pm 12 & $(4.9 \%)$ \\
\hline$A_{c}$ & -199 & \pm 18 & $(9.1 \%)$ \\
\hline \multicolumn{4}{|c|}{ Five ortho-molecules at $T=1.5 \mathrm{~K}$} \\
\hline$A_{V}$ & -80.0 & \pm 1.0 & $(1.3 \%)$ \\
\hline$A_{s}$ & 214 & \pm 5.2 & $(2.4 \%)$ \\
\hline$A_{c}$ & -154 & \pm 6.4 & $(4.1 \%)$ \\
\hline
\end{tabular}

component of the intermolecular interaction (the precise expressions are given in the paper [7]). The ratios of the rotational energies of the clusters with five and three ortho-molecules (for the same total number $N$ of molecules) at $T=1.5 \mathrm{~K}$ lie between 2.73 and 3.03. As functions of $N$, the rotational energies $E_{\text {rot }}=E_{\text {rot }}(N)$ at temperature $T=1.5 \mathrm{~K}$ exhibit, in each of the two cases (three ortho-molecules and five ortho-molecules in the cluster), five local minima at $N=13,19,23,26$, and 29, as well as three less pronounced minima at $N=$ 32,34 , and 37 . These eight values of $N$ coincide exactly with the positions of the local minima of the chemical potentials $\mu(N)$ at the same temperature (Fig. 2) and, apparently, are also of a geometric origin. As the temperature is raised, the minima of the function $E_{r o t}(N)$ smoothen.
The most important spatial characteristic of a cluster is its radial density profile $\rho(R)$, i.e. the number of particles per volume unit at a distance of $R$ to the center of mass. The function $\rho(R)$ satisfies the normalization

$$
\int_{0}^{\infty} \rho(R) R^{2} d R=\frac{N}{4 \pi},
$$

the mean values $\langle R\rangle$ and $\left\langle R^{2}\right\rangle$ of the distance of the particle from the cluster center of mass and of the square of this distance, respectively, being calculated by the formulas

$$
\langle R\rangle=\frac{4 \pi}{N} \int_{0}^{\infty} \rho(R) R^{3} d R, \quad\left\langle R^{2}\right\rangle=\frac{4 \pi}{N} \int_{0}^{\infty} \rho(R) R^{4} d R .
$$

In the case of clusters containing orthohydrogen molecules, we found the distributions $\rho(R)$ for the whole cluster $\left(\rho_{\text {all }}\right)$ and separately for the para-molecules $\left(\rho_{\text {para }}\right)$ and the ortho-molecules $\left(\rho_{\text {ortho }}\right)$. In these calculations, we employed the same normalization (4) for all the three profiles:

$$
\begin{gathered}
\int_{0}^{\infty} \rho_{\text {all }}(R) R^{2} d R=\int_{0}^{\infty} \rho_{\text {para }}(R) R^{2} d R \\
\quad=\int_{0}^{\infty} \rho_{\text {ortho }}(R) R^{2} d R=\frac{N}{4 \pi},
\end{gathered}
$$

which enabled us to compare directly the radial distributions of the para- and orthohydrogen molecules.

Of course, while computing the radial density profile $\rho(R)$, we did not assume the cluster to be spherically symmetric. It is also worthwhile to note that the function $\rho(R)$ of the scalar argument $R$ was determined directly, without finding first the spatial density (as a function of the vector argument $\mathbf{R}$ ) and its subsequent averaging over two angular variables.

As the calculations showed, the presence of several ortho-molecules does not affect significantly the radial density profile $\rho(R)=\rho_{\text {all }}(R)$ of the whole cluster, and this profile almost coincides with that of the pure parahydrogen cluster for the same $N$ and $T$. On the other hand, the shape of the function $\rho(R)$ varies very strongly with $N$ and $T$. For instance, at $T=1.5 \mathrm{~K}$, the density $\rho(R)$ decreases as $R \longrightarrow 0$ for $4 \leq N \leq 8$, makes a plateau or increases for $9 \leq N \leq 18$ (see Fig. 6 for $N=13$ ), decreases for $19 \leq N \leq 38$ (see Fig. 7 for $N=26$ and Fig. 8 for $N=38$ ), and increases again for $39 \leq N \leq 40$. These differences in the behavior of the radial density profile $\rho(R)$ for small $R$ values persist in a somewhat smoothened form as the temperature is raised to 3 or $4.5 \mathrm{~K}$. They agree with the data of many preceding works devoted to clusters $\left(\mathrm{H}_{2}\right)_{N}\left(\mathrm{D}_{2}\right)_{n}[1,3-$ $6,14,16-20,22,25-27]$. An increase in the density $\rho(R)$ as $R \longrightarrow 0$ is connected with the presence of a 


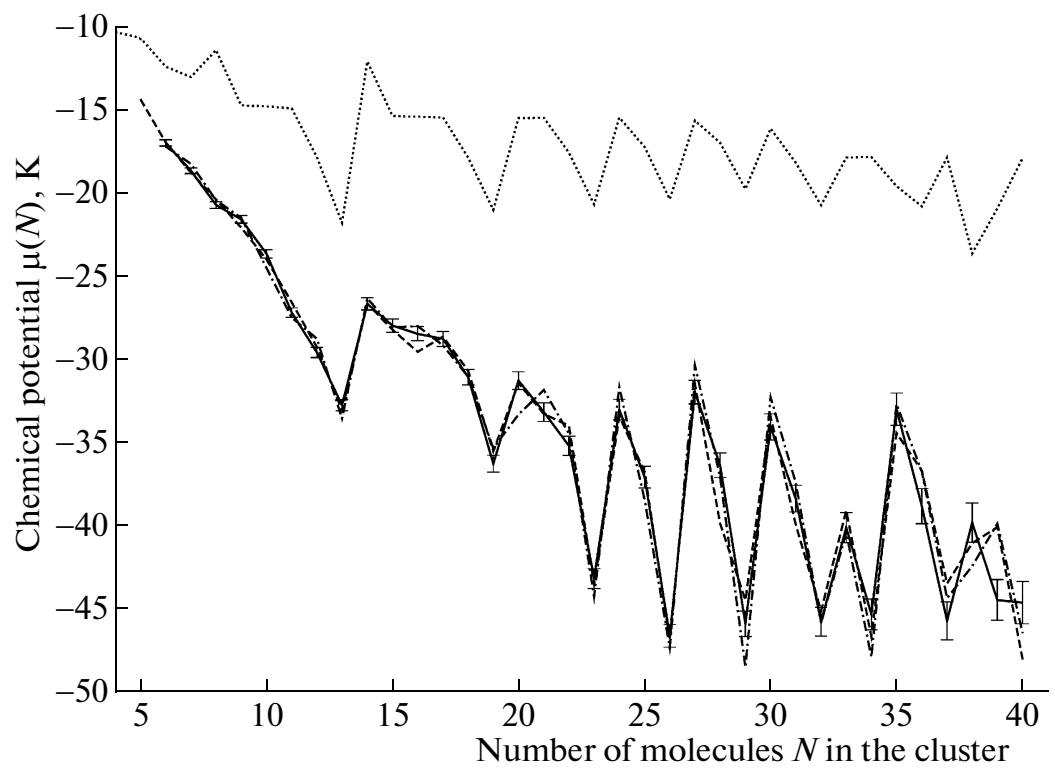

Fig. 2. The uppermost line represents quantities (3) divided by 10. The lower part of the figure presents the chemical potentials (2) of hydrogen clusters without ortho-molecules (the dashed line), with three ortho-molecules (the solid line), and with five orthomolecules (the dashed-and-dotted line) at temperature $T=1.5 \mathrm{~K}$. The standard deviations are shown only for the chemical potentials of the clusters $\left(\text { para }-\mathrm{H}_{2}\right)_{N-3}\left(\text { ortho }-\mathrm{H}_{2}\right)_{3}$; in the two other cases, the standard deviations are almost the same.

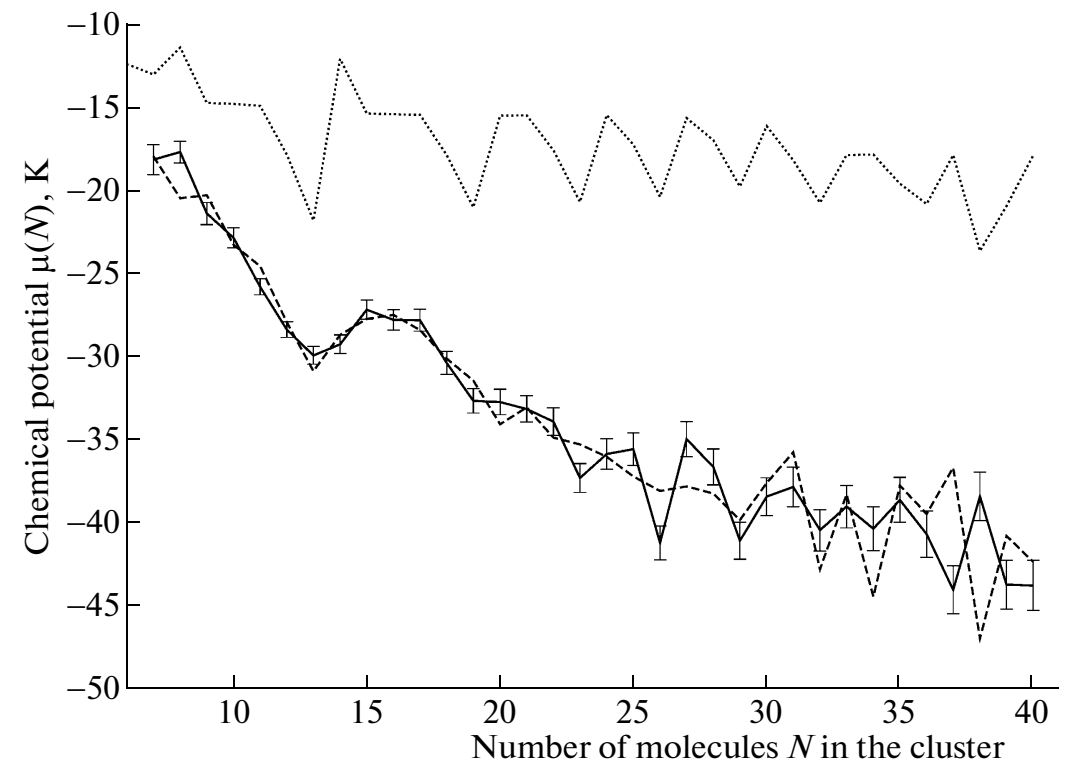

Fig. 3. The uppermost line represents quantities (3) divided by 10. The lower part of the figure presents the chemical potentials (2) of hydrogen clusters without ortho-molecules (the dashed line) and with three ortho-molecules (the solid line) at temperature $T=3 \mathrm{~K}$. The standard deviations are shown only for the chemical potentials of the clusters $\left(\text { para }-\mathrm{H}_{2}\right)_{N-3}\left(\text { ortho }-\mathrm{H}_{2}\right)_{3}$; in the case of the clusters $\left(\text { para }-\mathrm{H}_{2}\right)_{N}$, the standard deviations are almost the same.

central molecule in the cluster. In turn, the latter is also caused, in the long run, by the geometry of the energy-minimal configurations of classical particles with pairwise interaction $[46,47,52,53]$. The radial densities of the clusters $\left(\text { para }-\mathrm{H}_{2}\right)_{N}$ at zero absolute temperature for all the $N$ values from 3 to 50 are presented in the papers $[14,17,22]$.

On the other hand, an analysis of the radial density profiles $\rho_{\text {para }}(R)$ and $\rho_{\text {ortho }}(R)$ proves that in hydrogen clusters containing ortho-molecules, the latter exhibit 


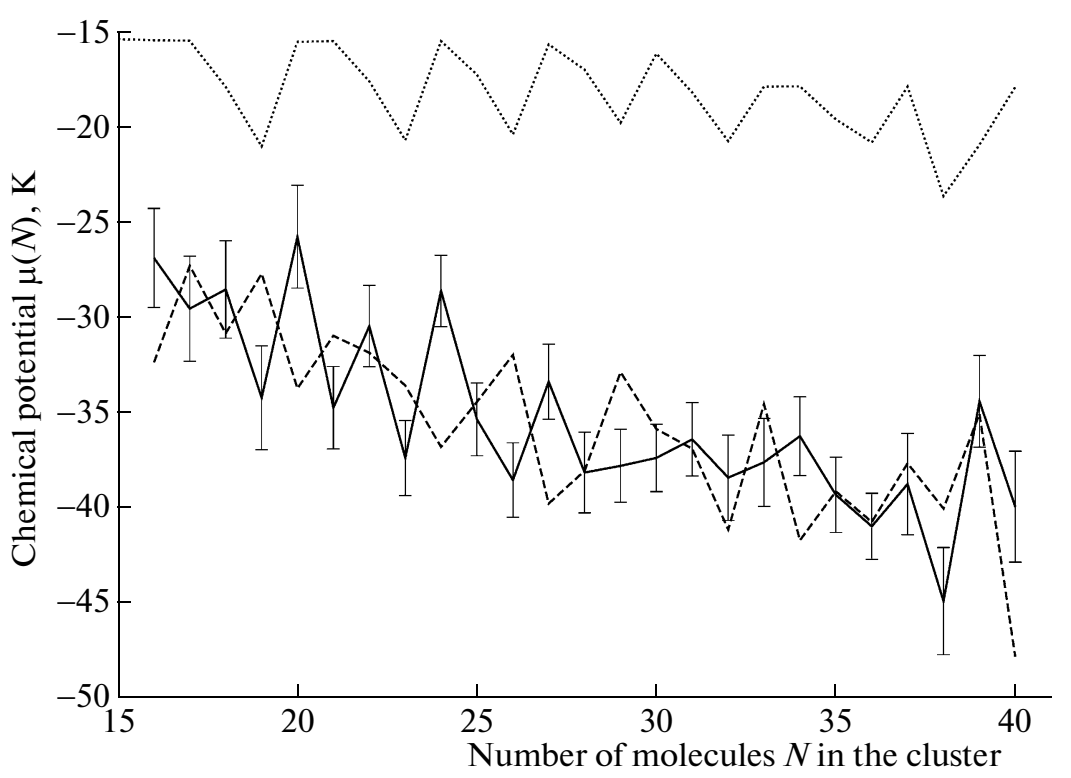

Fig. 4. The uppermost line represents quantities (3) divided by 10. The lower part of the figure presents the chemical potentials (2) of hydrogen clusters without ortho-molecules (the dashed line) and with three ortho-molecules (the solid line) at temperature $T=4.5 \mathrm{~K}$. The standard deviations are shown only for the chemical potentials of the clusters $\left(\text { para }-\mathrm{H}_{2}\right)_{N-3}\left(\text { ortho- } \mathrm{H}_{2}\right)_{3}$; in the case of the clusters $\left(\text { para }-\mathrm{H}_{2}\right)_{N}$, the standard deviations are almost the same.

a larger probability (compared to the para-molecules) to reside in the central region of the cluster and a smaller probability to be located near its surface. This partial spatial separation of the ortho- and para-components of the cluster is explained by the fact that the potential energy of the orthohydrogen molecule interaction is larger on the average (in absolute value) due

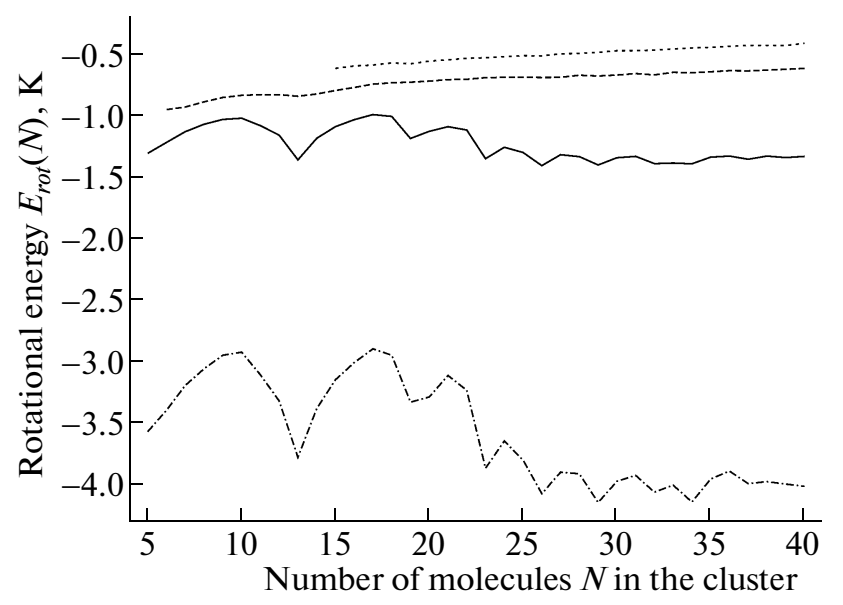

Fig. 5. The rotational energies of the clusters. The three upper lines correspond to clusters with three orthohydrogen molecules at temperatures $T=4.5,3$, and $1.5 \mathrm{~K}$ (from top to bottom). The standard deviations do not exceed a few millidegrees Kelvin. The lowermost line corresponds to the clusters $\left(\text { para }-\mathrm{H}_{2}\right)_{N-5}\left(\text { ortho }-\mathrm{H}_{2}\right)_{5}$ at temperature $T=1.5 \mathrm{~K}$. The standard deviations do not exceed one or two centidegrees Kelvin. to the presence of the anisotropic quadrupole-quadrupole term. The function $\rho_{\text {ortho }}(R)$ is larger than $\rho_{\text {all }}(R)$ for small distances $R$ (smaller than a certain value $R_{0}$ ), whereas $\rho_{\text {all }}(R)$ exceeds $\rho_{\text {ortho }}(R)$ for $R>R_{0}$ (the calculations show that the "critical" distance $R_{0}$ increases with $N)$. For the density profiles $\rho_{\text {para }}(R)$, the situation is opposite. Figures 6-8 present typical examples for $T=1.5 \mathrm{~K}$. In all the cases, the general behavior of the functions $\rho_{\text {para }}(R)$ and $\rho_{\text {ortho }}(R)$ is similar on the whole to the shape of the function $\rho_{\text {all }}(R)$. The radial separation of the ortho- and para-components of the cluster is enhanced as the number $m$ of ortho-molecules in the cluster increases (this effect is very clear in Figs. 68 ), the total number $N$ of molecules grows (within the ranges sampled in this work), and the temperature $T$ is lowered.

The trend of orthohydrogen molecules to concentrate in the central region of the cluster can be estimated by computing the ratio of the mean squares of the distances of the para- and ortho-molecules from the cluster center of mass:

$$
\frac{\left\langle R^{2}\right\rangle_{\text {para }}}{\left\langle R^{2}\right\rangle_{\text {ortho }}}=\left[\int_{0}^{\infty} \rho_{\text {ortho }}(R) R^{4} d R\right]_{0}^{-1} \int_{0}^{\infty} \rho_{\text {para }}(R) R^{4} d R
$$

The calculation results are presented in Fig. 9. This figure shows that ratio (5) exceeds unit in all the cases. Moreover, the higher the temperature, the smaller this ratio, as a whole. At $T=1.5 \mathrm{~K}$, ratio (5) is noticeably larger for the clusters with five ortho-molecules than 


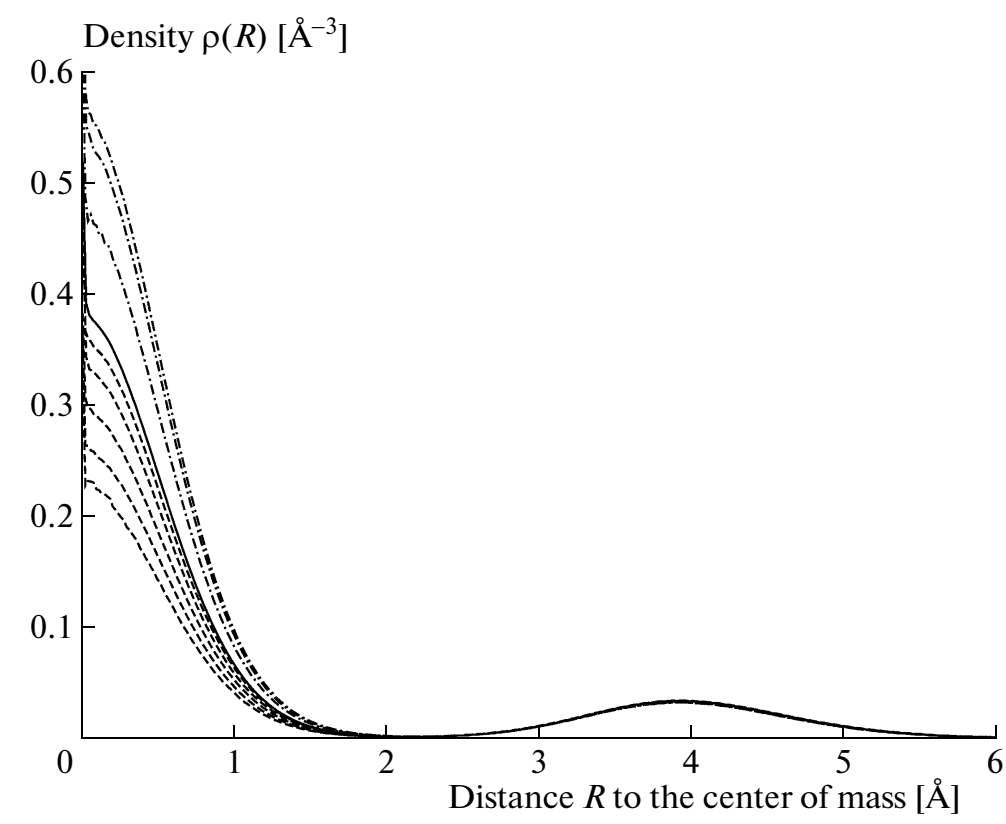

Fig. 6. The radial density profiles of clusters of 13 hydrogen molecules at temperature $T=1.5 \mathrm{~K}$ with various numbers $\mathrm{m}$ of orthomolecules. The nine lines presented correspond to the following functions (from top to bottom in the region $R \leq 1 \AA$ ). The three dashed-and-dotted lines show $\rho_{\text {ortho }}(R): m=4$ and 5 (the densities of ortho-molecules in these two cases are undistinguishable in the figure scale); $m=3$ and 6 (the densities of ortho-molecules in these two cases are also undistinguishable in the figure scale); $m=2$. The solid line shows $\rho(R)$ for $m=0$ and $\rho_{\text {all }}(R)$ for $2 \leq m \leq 6$ (all these six functions are undistinguishable in the figure scale). The five dashed lines show $\rho_{\text {para }}(R): m=2,3,4,5$, and 6 .

for the clusters with three ortho-molecules. On the whole, this ratio increases as the total number of molecules in the cluster rises. At the magic value $N=13$, ratio (5) at $T=1.5 \mathrm{~K}$ attains a local maximum for the clusters $\left(\text { para }-\mathrm{H}_{2}\right)_{\mathrm{N}-3}\left(\text { ortho }-\mathrm{H}_{2}\right)_{3}$ as well as for the clusters $\left(\text { para }-\mathrm{H}_{2}\right)_{N-5}\left(\text { ortho }-\mathrm{H}_{2}\right)_{5}$.

Of course, all the effects listed hold also for the ratios $\langle R\rangle_{\text {para }} /\langle R\rangle_{\text {ortho }}$ of the mean distances of the paraand ortho-molecules from the cluster center of mass. We have preferred to consider the ratios of the mean squares of the distances because in the framework of the liquid drop model, the quantity $\left\langle R^{2}\right\rangle$ is used more often than $\langle R\rangle$ (for instance, the so-called equivalent uniform radius of a drop is equal to $\left.\left(5\left\langle R^{2}\right\rangle / 3\right)^{1 / 2}[49]\right)$.

A heightened concentration of orthohydrogen molecules in the central region of the cluster agrees with the remarks of the work [29] as well as with the observations of the paper [7], where an analogous effect was noted for paradeuterium molecules in the cluster $\left(\text { ortho }-\mathrm{D}_{2}\right)_{10}\left(\text { para }-\mathrm{D}_{2}\right)_{3}$ at $T=1 \mathrm{~K}$. On the other hand, the data of the work [20] imply that the radial density profile $\rho_{\text {ortho }}(R)$ of the ortho-molecule in the cluster $\left(\text { para }-\mathrm{H}_{2}\right)_{24}\left(\text { ortho- } \mathrm{H}_{2}\right)_{1}$ at $T=1 \mathrm{~K}$ almost coincides with the radial density profile $\rho(R)$ for the pure parahydrogen cluster $\left(\text { para }-\mathrm{H}_{2}\right)_{25}$ at the same temperature. This does not contradict the conclusions of the present paper: the smaller is the number of ortho-molecules in a cluster, the weaker is the spatial separation of its ortho- and para-components. Moreover, since the interaction between a hydrogen ortho- molecule and a para-molecule is almost the same as the interaction between two para-molecules [7], the presence of a single ortho-molecule in a cluster cannot affect its structure at all.

Of course, the tendency of orthohydrogen molecules to be closer to the cluster center compared to parahydrogen molecules can be observed also while analyzing the distributions of the pairwise distances between molecules. For the distribution function $g(r)$ of pairwise distances $r$ between particles in a cluster, the optimal normalization is given by the equation [14]

$$
\int_{0}^{\infty} g(r) r^{2} d r=\frac{N-1}{4 \pi} .
$$

Figure 10 presents the ratios $\left\langle r^{2}\right\rangle_{\text {para }} /\left\langle r^{2}\right\rangle_{\text {ortho }}$ of the mean squares of the pairwise distances between orthomolecules and between para-molecules. The ratios are found by a formula analogous to (5). As one sees in the figure, these ratios exceed unit in all the cases and exhibit the same trends as ratios (5) of the mean squares of the distances of molecules from the cluster center of mass.

Our calculations also imply that the distribution of the angles in the triangles whose vertices coincide with the location of molecules in the cluster is determined only by the total number $N$ of molecules and does not depend on the temperature and the number of orthohydrogen molecules almost at all. 


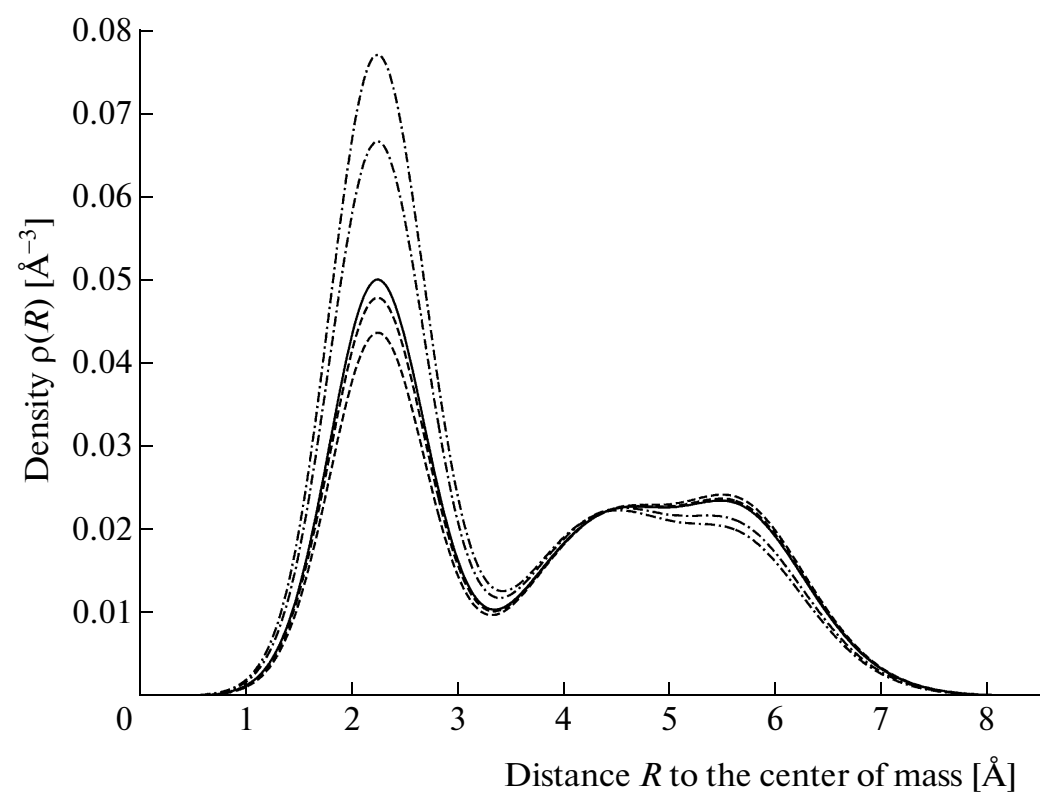

Fig. 7. The radial density profiles of clusters of 26 hydrogen molecules at temperature $T=1.5 \mathrm{~K}$ with various numbers $m$ of orthomolecules. The five lines presented correspond to the following functions (from top to bottom in the region of the left "hump": $R \approx 2.25 \AA$ ). The two dashed-and-dotted lines show $\rho_{\text {ortho }}(R): m=5$ and 3 . The solid line shows $\rho(R)$ for $m=0$ and $\rho_{\text {all }}(R)$ for $m=3$ and 5 (these three functions are undistinguishable in the figure scale). The two dashed lines show $\rho_{\text {para }}(R): m=3$ and 5 .

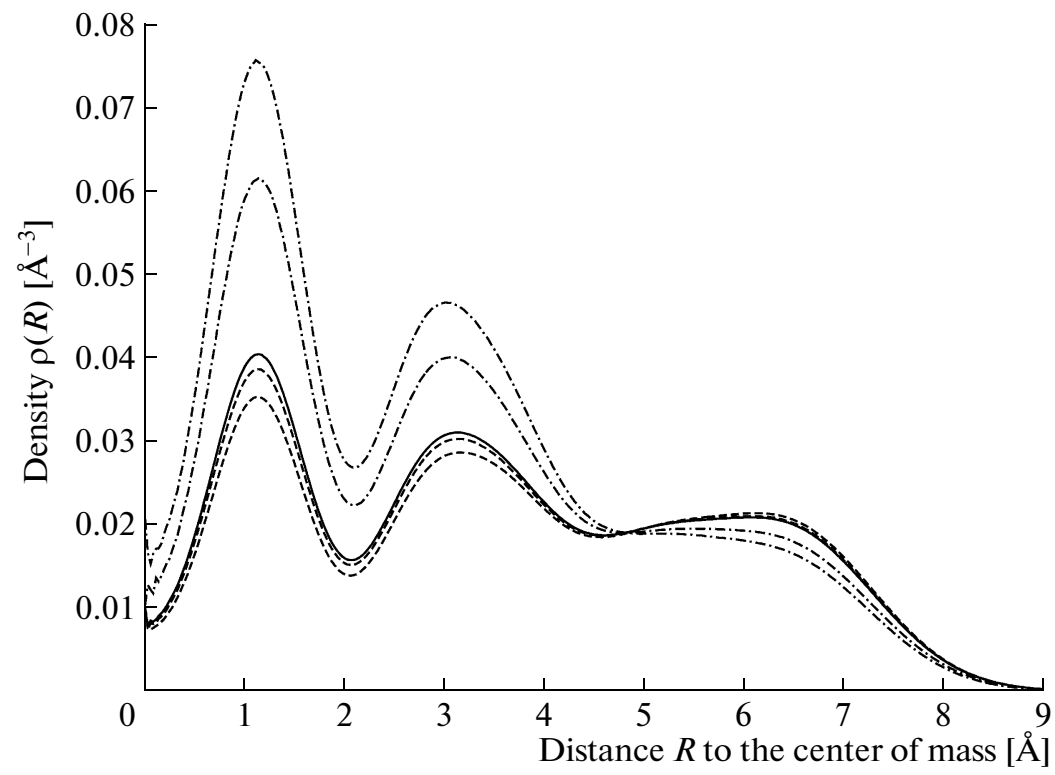

Fig. 8. The radial density profiles of clusters of 38 hydrogen molecules at temperature $T=1.5 \mathrm{~K}$ with various numbers $m$ of orthomolecules. The five lines presented correspond to the following functions (from top to bottom in the region of the two left "humps": $1 \AA \leq R \leq 3 \AA)$. The two dashed-and-dotted lines show $\rho_{\text {ortho }}(R): m=5$ and 3 . The solid line shows $\rho_{\text {all }}(R)$ for $m=3$ and 5 (these two functions are undistinguishable in the figure scale). The two dashed lines show $\rho_{\text {para }}(R): m=3$ and 5 .

The results of the present paper show that the presence of several ortho-molecules in a parahydrogen cluster affects slightly, as a whole, the energy and structural properties of the cluster. On the other hand, the relative density of orthohydrogen molecules turns out to be higher near the cluster center than near its surface, the magic numbers for the rotational energy and the chemical potential coinciding. These observations seem to be in no way connected with any special features of the Lennard-Jones potential for the inter- 


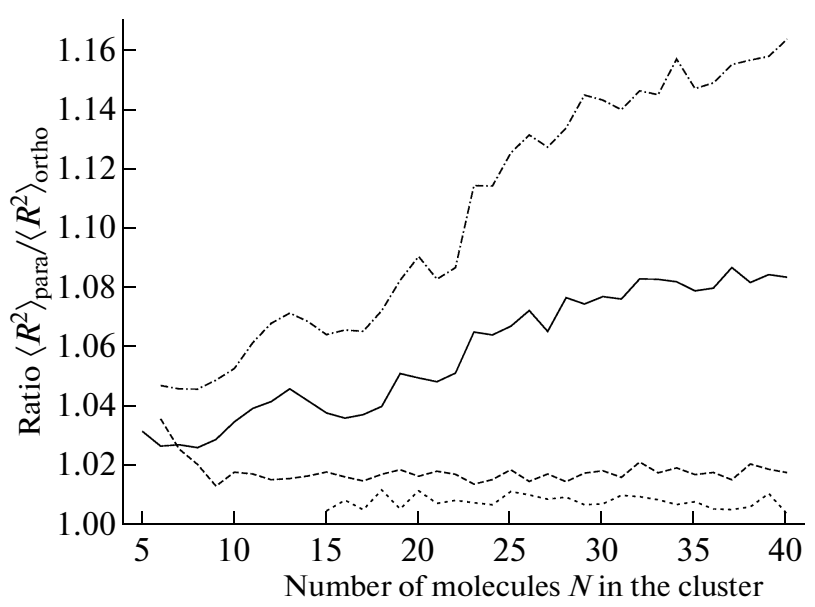

Fig. 9. Ratios (5) of the mean squares of the distances of hydrogen para- and ortho-molecules from the cluster center of mass. The uppermost line corresponds to the clusters $\left(\text { para }-\mathrm{H}_{2}\right)_{N-5}\left(\text { ortho }-\mathrm{H}_{2}\right)_{5}$ at temperature $T=1.5 \mathrm{~K}$. The three subsequent lines correspond to the clusters with three orthohydrogen molecules at temperatures $T=1.5,3$, and $4.5 \mathrm{~K}$ (from top to bottom).

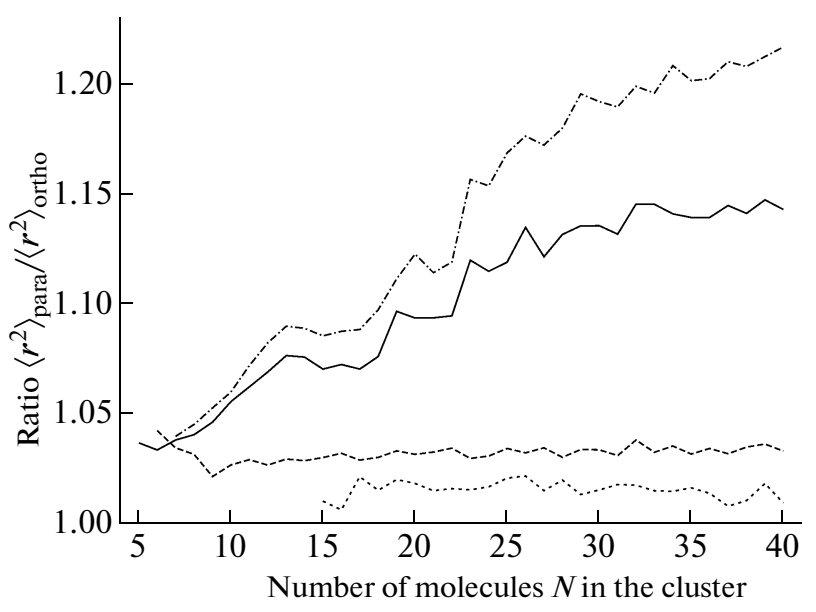

Fig. 10. The ratios $\left\langle r^{2}\right\rangle_{\text {para }} /\left\langle r^{2}\right\rangle_{\text {ortho }}$ of the mean squares of the pairwise distances between ortho-molecules and between para-molecules. The uppermost line corresponds to the clusters $\left(\text { para }-\mathrm{H}_{2}\right)_{N-5}\left(\text { ortho }-\mathrm{H}_{2}\right)_{5}$ at temperature $T=1.5 \mathrm{~K}$. The three subsequent lines correspond to the clusters with three orthohydrogen molecules at temperatures $T=1.5,3$, and $4.5 \mathrm{~K}$ (from top to bottom).

molecular interaction and, moreover, are expected to hold also if one takes into account more accurately the intermolecular exchange effects (in particular, superfluidity).

\section{ACKNOWLEDGMENTS}

The authors thank V. Buch who kindly provided to them computer programs for simulating hydrogen and deuterium clusters by the PIMC method. The codes used in this work were modernized versions of Buch's programs. The authors are grateful to O.A. Kornilov for interesting discussions of the results obtained.

\section{REFERENCES}

1. P. Sindzingre, D. M. Ceperley, and M. L. Klein, Phys. Rev. Lett. 67, 1871 (1991).

2. M. V. Rama Krishna and K. B. Whaley, Z. Phys. D 20, 223 (1991).

3. D. Scharf, G. J. Martyna, and M. L. Klein, Chem. Phys. Lett. 197, 231 (1992).

4. D. Scharf, M. L. Klein, and G. J. Martyna, J. Chem. Phys. 97, 3590 (1992).

5. M. A. McMahon, R. N. Barnett, and K. B. Whaley, J. Chem. Phys. 99, 8816 (1993).

6. M. A. McMahon and K. B. Whaley, Chem. Phys. 182, 119 (1994).

7. V. Buch, J. Chem. Phys. 100, 7610 (1994).

8. C. Chakravarty, Mol. Phys. 84, 845 (1995).

9. C. Chakravarty, Phys. Rev. Lett. 75, 1727 (1995).

10. C. Chakravarty, J. Chem. Phys. 104, 7223 (1996).

11. L. S. Costa and D. C. Clary, J. Chem. Phys. 117, 7512 (2002).

12. M. Carmichael, K. Chenoweth, and C. E. Dykstra, J. Phys. Chem. A 108, 3143 (2004).

13. G. Tejeda, J. M. Fernández, S. Montero, et al., Phys. Rev. Lett. 92, 223401 (2004).

14. J. E. Cuervo and P.-N. Roy, J. Chem. Phys. 125, 124314 (2006).

15. E. Rabani and J. Jortner, J. Phys. Chem. B 110, 18893 (2006).

16. F. Mezzacapo and M. Boninsegni, Phys. Rev. Lett. 97, 045301 (2006).

17. R. Guardiola and J. Navarro, Phys. Rev. A 74, 025201 (2006).

18. S. A. Khairallah, M. B. Sevryuk, D. M. Ceperley, and J. P. Toennies, Phys. Rev. Lett. 98, 183401 (2007).

19. F. Mezzacapo and M. Boninsegni, Phys. Rev. A 75, 033201 (2007).

20. F. Mezzacapo and M. Boninsegni, Phys. Rev. A 76, 021201 (2007).

21. J. I. Martínez, M. Isla, and J. A. Alonso, Eur. Phys. J. D 43, 61 (2007).

22. J. Navarro and R. Guardiola, J. Low Temp. Phys. 148, 857 (2007).

23. R. Guardiola and J. Navarro, Central Eur. J. Phys. 6 (1), 33 (2008).

24. R. Guardiola and J. Navarro, J. Chem. Phys. 128, 144303 (2008).

25. F. Mezzacapo and M. Boninsegni, Phys. Rev. Lett. 100, 145301 (2008).

26. J. Choo and Y. Kwon, J. Low Temp. Phys. 150, 358 (2008).

27. J. Choo and Y. Kwon, J. Korean Phys. Soc. 52, 259 (2008).

28. I. F. Silvera, Rev. Mod. Phys. 52, 393 (1980).

29. F. B. Buyvol-Kot, A. V. Kalinin, O. A. Kornilov, et al., Solid State Commun. 135, 532 (2005). 
30. J. Tang and A. R. W. McKellar, J. Chem. Phys. 121, 3087 (2004).

31. J. Tang and A. R. W. McKellar, J. Chem. Phys. 123, 114314 (2005).

32. D. M. Neumark, A. M. Wodtke, G. N. Robinson, et al., J. Chem. Phys. 82, 3045 (1985).

33. W. B. Chapman, B. W. Blackmon, S. A. Nizkorodov, and D. J. Nesbitt, J. Chem. Phys. 109, 9306 (1998).

34. L. Yu. Rusin and J. P. Toennies, Phys. Chem. Chem. Phys. 2, 501 (2000).

35. L. Yu. Rusin, M. B. Sevryuk, and J. P. Toennies, J. Chem. Phys. 122, 134314 (2005).

36. M. Ayabakan, M. Faubel, B. Martínez-Haya, et al., Chem. Phys. 229, 21 (1998).

37. L. Yu. Rusin, M. B. Sevryuk, U. Tappe, et al., Physicochemical Kinetics in Gas Dynamics (2007), vol. 5; http://www.chemphys.edu.ru/pdf/2007-06-21-001.pdf.

38. B. J. Berne and D. Thirumalai, Ann. Rev. Phys. Chem. 37, 401 (1986).

39. J. D. Doll, D. L. Freeman, and T. L. Beck, Adv. Chem. Phys. 78, 61 (1990).

40. D. M. Ceperley, Rev. Mod. Phys. 67, 279 (1995).

41. D. M. Ceperley and L. Mitas, Adv. Chem. Phys. 93, 1 (1996).
42. C. Chakravarty, Int. Rev. Phys. Chem. 16, 421 (1997).

43. I. F. Silvera and V. V. Goldman, J. Chem. Phys. 69, 4209 (1978).

44. U. Buck, F. Huisken, A. Kohlhase, et al., J. Chem. Phys. 78, 4439 (1983).

45. M. J. Norman, R. O. Watts, and U. Buck, J. Chem. Phys. 81, 3500 (1984).

46. D. J. Wales, J. P. K. Doye, A. Dullweber, et al., The Cambridge Cluster Database, http://www-wales.ch.cam. ac.uk/CCD.html.

47. D. J. Wales and J. P. K. Doye, J. Phys. Chem. A 101, 5111 (1997).

48. E. Krotscheck and R. Zillich, J. Chem. Phys. 115, 10161 (2001).

49. R. Guardiola, O. A. Kornilov, J. Navarro, and J. P. Toennies, J. Chem. Phys. 124, 084307 (2006).

50. V. L. Ginzburg and A. A. Sobyanin, Pis'ma Zh. Eksp. Teor. Fiz. 15, 343 (1972) [JETP Lett. 15, 242 (1972)].

51. F. Baletto and R. Ferrando, Rev. Mod. Phys. 77, 371 (2005).

52. B. M. Smirnov, Usp. Fiz. Nauk 167, 1169 (1997) [Phys. Usp. 40, 1117 (1997)].

53. B. M. Smirnov, Usp. Fiz. Nauk 177, 369 (2007) [Phys. Usp. 50, 354 (2007)]. 